\title{
ON SOME PASTING CYLINDERS ONTO A MANIFOLD WITH NEGATIVE (RICCI, SCALAR) CURVATURE ALONG COMPACT BOUNDARIES
}

\author{
By \\ Hajime KAWAKAMI
}

\section{Introduction.}

In Topology two manifolds are often pasted together. If both of these manifolds are riemannian, it is natural to consider the relation between geometry of the obtained manifold and geometry of original two manifolds. For this problem L.Z. Gao showed the following result [5]. If $M$ and $N$ are two complete riemannian manifolds with negative Ricci curvature of same dimension, then there is a complete riemannian metric on the connected sum $M \# N$ with negative Ricci curvature. Of course when he constructs this metric on $M \# N$, he changes both the metrics on $M$ and on $N$. Changing a point of view, we would like to take $M$ with boundary $\partial M$ and paste $M$ to another manifold with boundary along $\partial M$ not changing the metric of $M$. In this paper we show the following. Let $M$ be a negatively Ricci (resp. scalar) curved complete manifold with compact boundary and $\check{M}$ be a manifold without boundary obtained by pasting cylinders onto $M$. Then there is a complete metric on $\check{M}$ with negative Ricci (resp. scalar) curvature such that the inclusion $M \subset \check{M}$ is an isometric embedding and this metric is a warped product metric (cf. [2]) on $\check{M} \backslash S$ (where $S$ is an open neighborhood of $M$ ) whose vertical fibers are homothetic to $\partial M$. Moreover if we assume that $\partial M$ is totally geodesic in $M$, we have the same assertion as above for sectional curvature. Manifolds with metrics of this type are studied in some papers (e.g. [1], [4]).

We use the following notation for a $C^{\infty}$ manifold $X$ with or without boundary,

$\operatorname{Sec}_{-}(X):=\{$ all riemannian metrics on $X$ with negative sectional curvature,

$\operatorname{Ric}_{-}(X):=\{$ all riemannian metrics on $X$ with negative Ricci curvature $\}$,

Sca_ $(X):=\{$ all riemannian metrics on $X$ with negative scalar curvature $\}$.

Received May 18, 1989. Revised October 30, 1989. 
If $\partial X \neq \varnothing, " C^{\infty}$ " means that there exist a $C^{\infty}$ extension to an open manifold including $X$. Let $(M, g)$ be a riemannian manifold with compact connected $C^{\infty}$ boundary $\partial M$ and complete metric $g$, where the completeness means that any Cauchy sequence converges in $M$. We attach $\partial M \times(-\infty, \varepsilon)(\varepsilon>0$; small) to $M$ so that $\partial M \times[0, \varepsilon)$ is the $\varepsilon$-collar neighborhood and we denote by $\check{M}$ a $C^{\infty}$ manifold obtained in this way.

THEOREM. (1) We assume that $g \in \mathrm{Ric}_{-}(M)$ (resp. $\left.g \in \mathrm{Sca}_{-}(M)\right)$. Then there is a complete riemannian metric $\check{g}$ on $\check{M}$ such that $\check{g} \in \operatorname{Ric}_{-}(\check{M})\left(\right.$ resp. $\check{g} \in \operatorname{Sac}_{-}(\check{M})$ ), $\check{g}=g$ on $M$ and on $\check{M} \backslash S$ (where $S$ is an open neighborhood of $M$ ) $\check{g}$ is a warped product metric whose form is $d t \otimes d t+w^{2}(t) g_{\partial M}$ (where $g_{\partial M}$ is the restriction of $g$ to $\partial M)$. For any small $S$ we can construct $\check{g}$. Moreover $\operatorname{Sec}(\check{g})$ are negative on $\check{M} \backslash S$ and we can choose either $\check{g}$ that satisfies $\lim (\operatorname{Sec}(\check{g}))(x)=0,-1$ when $x$ goes to the infinite end of $\check{M} \backslash S$.

(2) We assume that $g \in \operatorname{Sec}_{-}(M)$ and $\partial M$ is totally geodesic in $M$. Then there is $\check{g} \in \operatorname{Sec}_{-}(\check{M})$ such that $\check{g}$ has properties stated in (1).

REMARK. The number of connected components of $\partial M$ in Theorem can be arbitrary. In fact, we can show it by applying the proof of Theorem to each component.

Acknowledgement. The author would like to express his thanks to Professor H. Kitahara and the referee for their useful suggestions.

\section{Lemmas and Proof of Theorem.}

First, in the following, we state two lemmas. Let $V$ be a $C^{\infty}$ manifold of $\operatorname{dim} V=m, \partial V=\varnothing$ or $\partial V \neq \varnothing$ and $W$ be a compact connected embedded $C^{\infty}$ submanifold of $V$ of $\operatorname{dim} W=k$. For a tubular neighborhood of $W$ in $V$ we choose coordinates $\left(y, t^{1}, \cdots, t^{n}\right)(y \in W, n=m-k)$ and we set

$$
U(W, \varepsilon):=\left\{x=\left(y, t^{1}, \cdots, t^{n}\right) \in V ;|t|^{2}:=\sum_{j=1}^{n}\left(t^{j}\right)^{2}<\varepsilon^{2}\right\} .
$$

Although $\left(y, t^{1}, \cdots, t^{n}\right)$ is local, we can assume that $|t|$ makes sense globally using the exponential map of the normal bundle of $W$ in $V$. In particular $U(\partial V, \varepsilon)$ is a collar neighborhood.

The following lemma is a direct generalization of Proposition 2.1 in [5].

LEMmA 1. Let $g_{0}, g_{1} \in \operatorname{Sec}_{-}(V)$ (resp. Ric $(V)$, Sca $\left.(V)\right)$ and $J^{1}\left(g_{0}\right)(x)=J^{1}\left(g_{1}\right)(x)$ for any point $x \in W$, where $J^{1}(\cdot)$ is the 1-jet. Then for any $\rho>0$ there are 
positive numbers $\rho_{1}, \rho_{2}$ and a metric $\bar{g}$ on $V$ such that $0<\rho_{2}<\rho_{1}<\rho, \bar{g} \in \operatorname{Sec}_{-}(V)$ (resp. $\left.\operatorname{Ric}_{-}(V), \mathrm{Sca}_{-}(V)\right)$ and

$$
\bar{g}(x)= \begin{cases}g_{0}(x) & \text { for any } x \in V \backslash U\left(W, \rho_{1}\right) \\ g_{1}(x) & \text { for any } x \in U\left(W, \rho_{2}\right) .\end{cases}
$$

Proof. It is similar to the proof of Proposition 2.1 in [5], so we state only an outline. We set $g_{s}:=(1-s) g_{0}+s g_{1}(0 \leqq s \leqq 1)$. Then

$$
J^{1}\left(g_{s}\right)(x)=J^{1}\left(g_{0}\right)(x)=J^{1}\left(g_{1}\right)(x) \quad \text { for any } x \in W
$$

and there is $\bar{\rho}>0$ such that

$$
g_{s} \in \operatorname{Sec}_{-}(U(W, \bar{\rho})) \quad\left(\text { resp. } \operatorname{Ric}_{-}(U(W, \bar{\rho})), \operatorname{Sca}_{-}(U(W, \bar{\rho})) .\right.
$$

Let $U=\left\{y=\left(y^{1}, \cdots, y^{k}\right)\right\}$ be a local coordinate chart of $W$. For $x \in U(U, \bar{\rho})$ we denote that $x=\left(x^{1}, \cdots, x^{m}\right)=\left(y^{1}, \cdots, y^{k}, t^{1}, \cdots, t^{n}\right)$ and $g_{s}(x)=\sum g_{\alpha \beta}(x, s) d x^{\alpha} d x^{\beta}$. We choose constants $\bar{\rho}, \lambda, \delta$ such that $0<\lambda^{1 / 2}=\delta \leqq \bar{\rho} / 4 \leqq 1 / 8$ and $\lambda$ is sufficiently small. Let $f$ be a $C^{\infty}$ function on $\boldsymbol{R}$ such that $0 \leqq f \leqq 1, f=1$ on $(-\infty, 1]$ and $f=0$ on $(2, \infty)$, and let $f_{\delta}(u):=f(u / \delta)$. We set $\Psi(y, t):=f_{\delta}\left(|t|^{\lambda}\right)$ for $(y, t) \in U(W, \bar{\rho})$ and $\Psi(x)=0$ for $x \in V \backslash U(W, \bar{\rho})$. Then $\Psi$ is a $C^{\infty}$ function on $V$. We set

$$
\bar{g}(x):=\sum g_{\alpha \beta}(x, \Psi(x)) d x^{\alpha} d x^{\beta} .
$$

We denote the Christoffel symbols of $g_{s}$ by $\Gamma_{\alpha \beta}^{r}(x, s)$ and we set

$$
\begin{aligned}
\Delta \Gamma_{\alpha \beta}^{\gamma}(x):= & (1 / 2) \Sigma_{\mu} g^{\gamma \mu}(x, s)\left\{\dot{g}_{\alpha \mu}(x, s) \cdot\left(\partial \Psi / \partial x^{\beta}\right)(x)\right. \\
& \left.+\dot{g}_{\beta \mu}(x, s) \cdot\left(\partial \Psi / \partial x^{\alpha}\right)(x)-\dot{g}_{\alpha \beta}(x, s) \cdot\left(\partial \Psi / \partial x^{\mu}\right)(x)\right\}\left.\right|_{s=\Psi(x),}
\end{aligned}
$$

where $\dot{g}_{\alpha \mu}(x, s):=\left(\partial g_{\alpha \mu} / \partial s\right)(x, s)$. Moreover we set

$$
\begin{aligned}
& A_{\beta \gamma \alpha}^{\xi}=A_{\beta \gamma \alpha}^{\xi}(x, s):=\dot{\Gamma}_{\alpha \beta}^{\xi} \cdot \partial \Psi / \partial x^{\gamma}-\dot{\Gamma}_{\gamma \beta}^{\xi_{\beta}} \cdot \partial \Psi / \partial x^{\alpha}+\partial\left(\Delta \Gamma_{\alpha \beta}^{\xi}\right) / \partial x^{\gamma}-\partial\left(\Delta \Gamma_{\gamma \beta}^{\xi}\right) / \partial x^{\alpha} \\
& +\Sigma_{\mu}\left\{\Gamma_{\gamma \mu}^{\xi} \Delta \Gamma_{\alpha \beta}^{\mu}+\left(\Delta \Gamma_{\gamma \mu}^{\xi}\right) \Gamma_{\alpha \beta}^{\mu}-\Gamma_{\alpha \mu}^{\xi} \Delta \Gamma_{\gamma \beta}^{\mu}-\left(\Delta \Gamma_{\alpha \mu}^{\xi}\right) \Gamma_{\gamma \beta}^{\mu}\right. \\
& \left.+\Delta \Gamma_{\gamma \mu}^{\xi} \cdot \Delta \Gamma_{\alpha \beta}^{\mu}-\Delta \Gamma_{\alpha \mu}^{\xi} \cdot \Delta \Gamma_{\gamma \beta}^{\mu}\right\}
\end{aligned}
$$

and

$$
A_{\alpha \beta}:=\Sigma_{\gamma} A_{\beta \gamma \alpha}^{\gamma} \text {. }
$$

Then we have the following from (1.1). For orthonormal (with respect to $\left.\bar{g}(x)=\left.g_{s}(x)\right|_{s=\Psi(x)}\right)$ vectors $X=\Sigma X^{\alpha} \partial / \partial x^{\alpha}, Y=\Sigma Y^{\alpha} \partial / \partial x^{\alpha} \in T_{x} V$

$$
\begin{aligned}
\operatorname{Sec}(\bar{g})(X, Y)= & \left.\operatorname{Sec}\left(g_{s}\right)(X, Y)\right|_{s=\Psi(x)} \\
& +\left.\sum Y^{\alpha} Y^{\beta} X^{\gamma} X^{\mu} A_{\beta \gamma \alpha}^{\xi}(x, s)\left(g_{s}(x)\right)_{\xi \mu}\right|_{s=\Psi(x)}
\end{aligned}
$$

and for a tangent vector $Z=\Sigma Z^{\alpha} \partial / \partial x^{\alpha} \in T_{x} V$ 


$$
\operatorname{Ric}(\bar{g})(Z, Z)=\left.\operatorname{Ric}\left(g_{s}\right)(Z, Z)\right|_{s=\Psi(x)}+\left.\sum Z^{\alpha} Z^{\beta} A_{\alpha \beta}(x, s)\right|_{s=\Psi(x)}
$$

and

$$
\operatorname{Sca}(\bar{g})(x)=\left.\operatorname{Sca}\left(g_{s}\right)(x)\right|_{s=\Psi(x)}+\left.\sum g_{s}^{\alpha \beta}(x) A_{\alpha \beta}(x, s)\right|_{s=\Psi(x)}
$$

where it holds that $\left.\left|A_{\beta \gamma \alpha}^{\xi}(x, s)\right|_{s=\Psi(x)}\left|\leqq C \lambda^{1 / 2}\right| t\right|^{\lambda}$ and $\left.\left|A_{\alpha \beta}(x, s)\right|_{s=\Psi(x)}\left|\leqq C \lambda^{1 / 2}\right| t\right|^{\lambda}$ for any $x=(y, t) \in U(W, \bar{\rho})$ ( $C$ is a positive constant). From (1.2), (1.4), (1.5) and (1.6) we can show Lemma 1.

LEMMA 2. Let $N$ be a compact $C^{\infty}$ manifold and $(N \times(-\infty, 0], g)$ a riemannian manifold with boundary $N \times\{0\}$. (We assume no condition about the sign of curvatures of $g$.) Then there is a complete riemannian metric $\hat{g}$ on $N \times(-\infty, 0]$ such that $\hat{g}$ is a solution of the partial differential inequality

$$
\operatorname{Ric}(\hat{g})<0 \quad \text { on } N \times(-\infty, 0]
$$

with the boundary condition

$$
J^{1}(\hat{g})(x)=J^{1}(g)(x) \quad \text { for any } x \in N \times\{0\} .
$$

Moreover if $N \times\{0\}$ is totally geodesic, there is a complete riemannian metric $\hat{g}$ on $N \times(-\infty, 0]$ such that

$$
\operatorname{Sec}(\hat{g})<0 \quad \text { on } N \times(-\infty, 0]
$$

with the same boundary condition as above. $\hat{g}$ is constructed concretely in the proof and satisfies the properties related in Theorem.

Proof. First we consider the case of Ricci curvature. We can assume that in a neighborhood of $N \times\{0\}$ the coordinate $t \in(-\infty, 0]$ is the arclength parameter of the geodesics normal to $N \times\{0\}$ with respect to $g$. Using this coordinate we define a symmetric tensor

$$
\hat{g}(y, t):=d t \otimes d t+(1+\Phi(t))^{2} g_{0}(y)+\rho(t) h(y)
$$

where $(y, t) \in N \times(-\infty, 0], g_{0}:=\left.g\right|_{N \times(0)}, h(y) \partial / \partial t$ is the 2nd fundamental form of $N \times\{0\}$ with respect to $g$ and $\Phi, \rho$ are $C^{\infty}$ functions. Because Ricci curvature is an average we choose $\Phi, \rho$ so that they gain enough negativity along the $t$-axis. For example it is as follows. Let $C$ and $\delta$ be positive constants. We set $\Phi(t):=\delta^{-3} C t^{2}$ and $\rho(t):=-2 t \exp (-1 /(t+2 \delta)+1 / 2 \delta)(t>-2 \delta)$, $:=0(t \leqq-2 \delta)$. supp $\rho \subset[-2 \delta, 0]$, so $\hat{g}$ is a warped product metric (cf. [2]) on $N \times(-\infty,-2 \delta)$. By a straightforward calculation (related after) we can show that $\hat{g}$ defined by $(2.1)$ is the desired metric $\left(\lim _{t \rightarrow-\infty}(\operatorname{Sec}(\hat{g}))(y, t)=0\right)$ if $C$ is sufficiently large and $\delta$ is sufficiently small. Moreover outside of supp $\rho$ we 
can deform $\hat{g}$ to the metric $\hat{g}^{\prime}(y, t):=d t \otimes d t+\cosh ^{2} t \cdot g_{0}(y)$ by slow degrees along $t$ preserving $\operatorname{Ric}<0$. This new metric $\hat{g}^{\prime}$ satisfies $\lim _{t \rightarrow-\infty}\left(\operatorname{Sec}\left(\hat{g}^{\prime}\right)\right)(y, t)=-1$.

We investigate 1-jets of $g$ and $\hat{g}$ and estimate of Ricci curvature of $\hat{g}$. At first, for $\Phi$ and $\rho$ we assume only that $\Phi$ and $\rho$ are nonnegative $C^{\infty}$ functions on $(-\infty, 0]$ and $\rho$ is so small that $\hat{g}$ is a metric. Let $U$ be a small open subset of $N$ and $\left(u^{1}, \cdots, u^{n}\right)$ be a system of $C^{\infty}$ orthonormal forms over $U$ with respect to $g_{0}$. By $H(y)$ we denote the matrix representation of $h(y)$ with respect to $\left(u^{1}, \cdots, u^{n}\right)$. We consider the $C^{\infty}$ function

$$
f: U \times \boldsymbol{R} \rightarrow \boldsymbol{R},(y, \lambda) \rightarrow \operatorname{det}(H(y)-\lambda I)
$$

and arrange solutions (i. e. principal curvatures) $\lambda(y)$ of $f(y, \lambda)=0 ; \lambda_{1}(y) \leqq$ $\cdots \leqq \lambda_{n}(y)$. We set

$U_{0}:=\left\{y \in U\right.$; there is a neighborhood $V_{y}$ of $y$ such that $\lambda_{1}(z), \cdots, \lambda_{n}(z)$ satisfy the same equalities and inequalities for all $z$ in $\left.V_{y}\right\}$.

By the Implicit Function Theorem we can show that each $\lambda_{j}$ is $C^{\infty}$ on $U_{0}$. Therefore there is a system of $C^{\infty}$ orthonormal forms $\left(e^{1}, \cdots, e^{n}\right)$ over $U_{0}$ with respect to $g_{0}$ such that $h(y)=\Sigma \lambda_{\alpha}(y) e^{\alpha}(y) \otimes e^{\alpha}(y)$ for $y \in U_{0}$. On the other hand using continuity of each $\lambda_{j}$ (cf. [3] pp.76-77) we can show that $U_{0}$ is open dense in $U$. Summing up the above we have that

$$
\hat{g}(y, t)=d t \otimes d t+(1+\Phi(t))^{2} \sum e^{\alpha}(y) \otimes e^{\alpha}(y)+\rho(t) \sum \lambda_{\alpha}(y) e^{\alpha}(y) \otimes e^{\alpha}(y)
$$

for $(y, t) \in \tilde{U}:=U_{0} \times(-\infty, 0]$ and $U_{0}$ is open dense in $U$. Let $\left(e_{1}, \cdots, e_{n}\right)$ be the dual orthonormal fields of $\left(e^{1}, \cdots, e^{n}\right)$ with respect to $g_{0}$ and $\left(v_{1}, \cdots, v_{n}, \partial / \partial t\right)$ be the orthonormal fields over $\tilde{U}$ such that $v_{\alpha}(y, t)=\Psi_{\alpha}^{-1}(y, t) e_{\alpha}(y)$ where $\Psi_{\alpha}(y, t):=\left\{(1+\Phi(t))^{2}+\rho(t) \lambda_{\alpha}(y)\right\}^{1 / 2}$.

We assume that $\Phi(0)=\Phi^{\prime}(0)=\rho(0)=0, \rho^{\prime}(0)=-2$. Then by a straightforward calculation we can show that on $U_{0} \times\{0\}$

$$
\begin{aligned}
& \partial g(\partial / \partial t, \partial / \partial t) / \partial t=\partial \hat{g}(\partial / \partial t, \partial / \partial t) / \partial t=0, \\
& \partial g\left(\partial / \partial t, e_{\alpha}\right) / \partial t=\partial \hat{g}\left(\partial / \partial t, e_{\alpha}\right) / \partial t=0, \\
& e_{\beta} g\left(\partial / \partial t, e_{\alpha}\right)=e_{\beta} \hat{g}\left(\partial / \partial t, e_{\alpha}\right)=0, \\
& e_{\alpha} g(\partial / \partial t, \partial / \partial t)=e_{\alpha} \hat{g}(\partial / \partial t, \partial / \partial t)=0, \\
& \partial g\left(e_{\alpha}, e_{\beta}\right) / \partial t=\partial \hat{g}\left(e_{\alpha}, e_{\beta}\right) / \partial t=-2 \delta_{\alpha \beta} \lambda_{\alpha}, \\
& e_{\gamma} g\left(e_{\alpha}, e_{\beta}\right)=e_{\gamma} \hat{g}\left(e_{\alpha}, e_{\beta}\right) .
\end{aligned}
$$

So we have that $J^{1}(g)=J^{1}(\hat{g})$ on $U_{0} \times\{0\}$, therefore, on $N \times\{0\}$. 
Next we consider the estimate of $\operatorname{Ric}(\hat{g})$. Let $\Phi$ and $\rho$ be any nonnegative $C^{\infty}$ functions again. We denote by $\hat{\nabla}$ the covariant derivative with respect to $\hat{g}$ and by $\hat{R}$ the curvature tensor of $\hat{\nabla}$. Let $X=\Sigma X^{k} v_{k}+X^{0} \partial / \partial t$ be a tangent vector on $\tilde{U}$. Then we have

$$
\begin{aligned}
\operatorname{Ric}(\hat{g})(X, X)= & \left(X^{0}\right)^{2} \sum_{\alpha=1}^{n} \hat{g}\left(\hat{R}_{v_{\alpha} \partial / \partial t} \partial / \partial t, v_{\alpha}\right) \\
& +\sum_{k=1}^{n}\left(X^{k}\right)^{2} \hat{g}\left(\hat{R}_{v_{k} \partial / \partial t} \partial / \partial t, v_{k}\right) \\
& +\sum_{k, \alpha=1}^{n}\left(X^{k}\right)^{2} \hat{g}\left(\hat{R}_{v_{\alpha} v_{k}} v_{k}, v_{\alpha}\right) \\
& +2 X^{0} \sum_{k, \alpha=1}^{n} X^{k} \hat{g}\left(\hat{R}_{v_{\alpha} \partial / \partial t} v_{k}, v_{\alpha}\right) \\
& +\sum_{j, k, \alpha=1, j \neq k}^{n} X^{j} X^{k} \hat{g}\left(\hat{R}_{v_{\alpha} v_{j}} v_{k}, v_{\alpha}\right) .
\end{aligned}
$$

We compute the right-hand side of (2.2), We have

$$
\begin{aligned}
& \hat{\nabla}_{\partial / \partial t} \partial / \partial t=0, \quad \hat{\nabla}_{\partial / \partial t} v_{\alpha}=0, \\
& \hat{g}\left(\hat{\nabla}_{v_{\alpha}} \partial / \partial t, \partial / \partial t\right)=0, \\
& \hat{g}\left(\hat{\nabla}_{v_{\alpha}} \partial / \partial t, v_{\beta}\right)=\delta_{\alpha \beta} \Psi_{\alpha}^{-1} \partial \Psi_{\alpha} / \partial t, \\
& \hat{g}\left(\hat{\nabla}_{v_{\alpha}} v_{\beta}, \partial / \partial t\right)=-\delta_{\alpha \beta} \Psi_{\alpha}^{-1} \partial \Psi_{\alpha} / \partial t, \\
& \hat{g}\left(\hat{\nabla}_{v_{\alpha}} v_{\beta}, v_{\gamma}\right)=-\frac{1}{2} \delta_{\alpha \beta} \Psi_{\gamma}^{-1} \Psi_{\alpha}^{-2} \rho e_{\gamma} \lambda_{\alpha}+\frac{1}{2} \delta_{\alpha \gamma} \Psi_{\bar{\beta}^{-1}}^{-1} \Psi_{\alpha}^{-2} \rho e_{\beta} \lambda_{\alpha} \\
& \quad+\Psi_{\alpha}^{-1} \Psi_{\bar{\beta}}^{-1} \Psi_{\gamma}^{-1}(1+\Phi)^{2} g\left(D_{e_{\alpha}} e_{\beta}, e_{\gamma}\right)+\Psi_{\alpha}^{-1} \Psi_{-\bar{\beta}}^{1} \Psi_{\gamma}^{-1} \rho G_{\alpha \beta \gamma},
\end{aligned}
$$

where $D$ is the covariant derivative with respect to $g_{0}$ and

$$
\begin{aligned}
G_{\alpha \beta \gamma}(y):= & (1 / 2)\left\{\lambda_{\gamma}(y) g\left(\left[e_{\alpha}, e_{\beta}\right], e_{\gamma}\right)+\lambda_{\beta}(y) g\left(\left[e_{\gamma}, e_{\alpha}\right], e_{\beta}\right)\right. \\
& \left.-\lambda_{\alpha}(y) g\left(e_{\alpha},\left[e_{\beta}, e_{\gamma}\right]\right)\right\} .
\end{aligned}
$$

By (2.3) we have the following.

$$
\begin{aligned}
\hat{g}\left(\hat{R}_{v_{\alpha} \partial / \partial t} \partial / \partial t, v_{\alpha}\right)= & -\Psi_{\alpha}^{-2}\left\{(1+\Phi) \Phi^{\prime \prime}+\left(\Phi^{\prime}\right)^{2}\right\} \\
& -\frac{1}{2} \Psi_{\alpha}^{-2} \rho^{\prime \prime} \lambda_{\alpha}+\frac{1}{4}\left\{\Psi_{\alpha}^{-2} \partial\left(\Psi_{\alpha}^{2}\right) / \partial t\right\}^{2}
\end{aligned}
$$

This term is an essential one. Next for $\alpha \neq k$

$$
\begin{aligned}
& \hat{g}\left(\hat{R}_{v_{\alpha} \partial / \partial t} v_{k}, v_{\alpha}\right) \\
& =\frac{1}{4} \Psi_{k}^{-1} \Psi_{\alpha}^{-2} e_{k} \lambda_{\alpha}\left[\Psi_{k}^{-2} \rho\left\{\partial\left(\Psi_{k}^{2}\right) / \partial t\right\}+\Psi_{\alpha}^{-2} \rho\left\{\partial\left(\Psi_{\alpha}^{2}\right) / \partial t\right\}-2 \rho^{\prime}\right] \\
& +\frac{1}{2} \Psi_{k}^{-1} \Psi_{\alpha}^{-2}(1+\Phi)\left[\Psi_{k}^{-2}(1+\Phi)\left\{\partial\left(\Psi_{k}^{2}\right) / \partial t\right\}\right. \\
& \left.\quad+\Psi_{\alpha}^{-2}(1+\Phi)\left\{\partial\left(\Psi_{\alpha}^{2}\right) / \partial t\right\}-4 \Phi^{\prime}\right] g\left(D_{e_{\alpha}} e_{k}, e_{\alpha}\right) \\
& +\frac{1}{2} \Psi_{k}^{-1} \Psi_{\alpha}^{-2} G_{\alpha k \alpha}\left[\Psi_{k}^{-2} \rho\left\{\partial\left(\Psi_{k}^{2}\right) / \partial t\right\}+\Psi_{\alpha}^{-2} \rho\left\{\partial\left(\Psi_{\alpha}^{2}\right) / \partial t\right\}-2 \rho^{\prime}\right]
\end{aligned}
$$


$\hat{g}\left(\hat{R}_{v_{\alpha} v_{k}} v_{k}, v_{\alpha}\right)$ and $\hat{g}\left(\hat{R}_{v_{\alpha} v_{j}} v_{k}, v_{\alpha}\right)(j \neq k)$ are more complicated, but we can estimate them easily. So we only write the explicit form of $\hat{g}\left(\hat{R}_{v_{\alpha} v_{k}} v_{k}, v_{\alpha}\right)$ for reader's information in Appendix. Now we set $\Phi(t):=\delta^{-3} C t^{2}$ and $\rho(t):=-2 t \exp (-1 /(t+2 \delta)+1 / 2 \delta)(t>-2 \delta),:=0(t \leqq-2 \delta)$ and we estimate the right-hand side of $(2.2)$, Depending on a constant $\xi(0<\xi<1)$ if we choose sufficiently small $\delta$, we have that

$$
\begin{aligned}
& -\Psi_{\alpha}^{-2}\left\{\xi(1+\Phi) \Phi^{\prime \prime}+\left(\Phi^{\prime}\right)^{2}\right\}-\frac{1}{2} \Psi_{\alpha}^{-2} \rho^{\prime \prime} \lambda_{\alpha} \\
& +\frac{1}{4}\left\{\Psi_{\alpha}^{-2} \partial\left(\Psi_{\alpha}^{2}\right) / \partial t\right\}^{2}<-\frac{1}{2} \xi C \delta^{-3}\left(1+C \delta^{-3} t^{2}\right) .
\end{aligned}
$$

So we have that for any unit tangent vector $X$ on $\tilde{U}$.

$$
\begin{aligned}
& \frac{1-\xi}{n+1} \Psi_{\bar{\beta}^{2}}^{-2}(1+\Phi) \Phi^{\prime \prime}+\left(X^{0}\right)^{2} \sum_{\alpha=1}^{n} \hat{g}\left(\hat{R}_{v_{\alpha} \partial / \partial t} \partial / \partial t, v_{\alpha}\right) \\
& \quad+\sum_{k=1}^{n}\left(X^{k}\right)^{2} \hat{g}\left(\hat{R}_{v_{k} \partial / \partial t} \partial / \partial t, v_{k}\right)<0,
\end{aligned}
$$

where $\beta$ is so choosen that $\left(X^{\beta}\right)^{2}=\max _{\alpha=0, \ldots, n}\left(X^{\alpha}\right)^{2} \geqq 1 /(n+1)$. On the other hand we can estimate the $3 \mathrm{rd}, 4$ th, 5 th terms of the right-hand side of $(2.2)$ by $(n+1)^{-1}(1-\xi) \Psi_{\bar{\beta}}^{-2}(1+\Phi) \Phi^{\prime \prime}$ using $(2.5)$ and certain explicit forms of $\hat{g}\left(\hat{R}_{v_{\alpha} v_{k}} v_{k}, v_{\alpha}\right)$ (cf. Appendix) and $\hat{g}\left(\hat{R}_{v_{\alpha} v_{j}} v_{k}, v_{\alpha}\right)(j \neq k)$. Therefore we have that $\operatorname{Ric}(\hat{g})(X, X)<0$ for any unit tangent vector $X$ on $\tilde{U}$, that is, $\operatorname{Ric}(\hat{g})<0$ on $\tilde{U}$. This inequality $\operatorname{Ric}(\hat{g})<0$ can be extended to $U \times(-\infty, 0]$ because that $U_{0}$ is open dense in $U$. Here, by (2.6), we can show that not only "@” but also " $<$ " holds. After all we have $\operatorname{Ric}(\hat{g})<0$ on $N \times(-\infty, 0]$ because that we can choose the universal constants $\delta, C$ by the compactness of $N$.

Next we consider the case of sectional curvature. We assume that $N \times\{0\}$ is totally geodesic. Then we find

$$
\hat{g}(y, t):=d t \otimes d t+(1+\Phi(t))^{2} g_{0}(y)
$$

is the desired metric. In fact $J^{1}(\hat{g})=J^{1}(g)$ on $N \times\{0\}$ the same as the case of Ricci curvature and $\operatorname{Sec}(\hat{g})<0$ on $N \times(-\infty, 0]$ by a formula in [2] p. 27. //

Now we show Theorem by using above two lemmas.

PROOF OF THEOREM. We attach $\partial M \times(-\varepsilon, \varepsilon)$ to $M$ so that $\partial M \times[0, \varepsilon)$ is the $\varepsilon$-collar with respect to $g$ and we denote by $\tilde{M}$ the $C^{\infty}$ manifold obtained by this way. We take small $\varepsilon$ so that we can extend $g$ to a metric $\tilde{g}$ on $\tilde{M}$ with $\tilde{g} \in \operatorname{Ric}_{-}(\tilde{M})$ (resp. Sca- $(\tilde{M})$ ). We can assume that the above attaching map $\varphi: \partial M \times(-\varepsilon, \varepsilon) \hookrightarrow \tilde{M}, \quad(y, t) \rightarrow \varphi(y, t)$ is an injective diffeomorphism such that $t$ is the arclength parameter of the geodesics normal to $\varphi(\partial M \times\{0\})=\partial M$ 
with respect to $\tilde{g}$. By Lemma 2 there is a metric $\hat{g}$ on $\partial M \times(-\infty, 0]$ such that $\hat{g} \in \mathrm{Ric}_{-}(\partial M \times(-\infty, 0])$ and $J^{1}(\hat{g})=J^{1}\left(\varphi^{*}(\tilde{g})\right)$ on $\partial M \times\{0\}$. We attach $\partial M \times(-\infty, 0]$ to $\tilde{M}$ by the above map $\varphi: \partial M \times(-\varepsilon, 0] \subset \tilde{M}$. The $C^{\infty}$ manifold obtained by this way is $\check{M}$. On $\varphi(\partial M \times(-\varepsilon, 0]) \subset \check{M}$ two metrics $\varphi_{*}(\hat{g})$ and $\tilde{g}$ are defined and they satisfy that $J^{1}\left(\varphi_{*}(\hat{g})\right)=J^{1}(\tilde{g})$ on $\varphi(\partial M \times\{0\})=\partial M$ and $\varphi_{*}(\hat{g})$, $\tilde{g} \in \operatorname{Ric}_{-}(\varphi(\partial M \times(-\varepsilon, 0]))$ (resp. Sca $\left.(\varphi(\partial M \times(-\varepsilon, 0]))\right)$. Therefore we can obtain the desired metric $\check{g}$ by Lemma 1 .

APPENDIX. An explicit form of $\hat{g}\left(\hat{R}_{v_{\alpha} v_{k}} v_{k}, v_{\alpha}\right)$.

We denote by $R$ the curvature tensor of $D$. Other notations are the same as above.

$$
\begin{aligned}
\hat{g}\left(\hat{R}_{v_{\alpha} v_{k}} v_{k}, v_{\alpha}\right) & \\
=- & \frac{1}{4} \Psi_{\alpha}^{-2} \Psi_{k}^{-2}\left\{\partial\left(\Psi_{\alpha}^{2}\right) / \partial t\right\}\left\{\partial\left(\Psi_{k}^{2}\right) / \partial t\right\}+\Psi_{\alpha}^{-2} \Psi_{k}^{-2}(1+\Phi)^{2} g\left(R_{e_{\alpha} e_{k}} e_{k}, e_{\alpha}\right)+\Psi_{\alpha}^{-2} \Psi_{k}^{-2} \rho \\
& \times\left[\sum_{i=1}^{n} \lambda_{i}\left\{\frac{1}{4} \Psi_{i}^{-2}\left((1+\Phi)^{2}-\rho \lambda_{i}\right)+1\right\} g\left(\left[e_{\alpha}, e_{k}\right], e_{i}\right)^{2}\right. \\
& +\sum_{i=1}^{n} \Psi_{i}^{-2}\left\{(1+\Phi)^{2} \lambda_{i}-\rho \lambda_{\alpha} \lambda_{k}\right\} g\left(\left[e_{i}, e_{\alpha}\right], e_{\alpha}\right) g\left(\left[e_{i}, e_{k}\right], e_{k}\right) \\
& -\frac{1}{4}(1+\Phi)^{2} \sum_{i=1}^{n} \Psi_{i}^{-2} \lambda_{i}\left\{g\left(\left[e_{i}, e_{\alpha}\right], e_{k}\right)+g\left(\left[e_{i}, e_{k}\right], e_{\alpha}\right)\right\}^{2} \\
& +\frac{1}{4} \rho \sum_{i=1}^{n} \Psi_{i}^{-2}\left\{\lambda_{k} g\left(\left[e_{i}, e_{\alpha}\right], e_{k}\right)+\lambda_{\alpha} g\left(\left[e_{i}, e_{k}\right], e_{\alpha}\right)\right\}^{2} \\
& -\lambda_{\alpha} \sum_{i=1}^{n} g\left(\left[e_{\alpha}, e_{k}\right], e_{i}\right) g\left(\left[e_{i}, e_{k}\right], e_{\alpha}\right) \\
& \left.-\lambda_{k} \sum_{i=1}^{n} g\left(\left[e_{\alpha}, e_{k}\right], e_{i}\right) g\left(\left[e_{\alpha}, e_{i}\right], e_{k}\right)\right] \\
+ & \frac{1}{4} \Psi_{\alpha}^{-2} \Psi_{k}^{-2} \rho^{2}\left[\left(\Psi_{\alpha}^{-1} e_{k} \lambda_{\alpha}\right)^{2}+\left(\Psi_{k}^{-1} e_{\alpha} \lambda_{k}\right)^{2}+\Psi_{\alpha}^{-2}\left(e_{\alpha} \lambda_{\alpha}\right)\left(e_{\alpha} \lambda_{k}\right)+\Psi_{k}^{-2}\left(e_{k} \lambda_{k}\right)\left(e_{k} \lambda_{\alpha}\right)\right] \\
- & \frac{1}{4} \Psi_{\alpha}^{-2} \Psi_{k}^{-2} \rho^{2} \sum_{i=1}^{n} \Psi_{i}^{-2}\left(e_{i} \lambda_{\alpha}\right)\left(e_{i} \lambda_{k}\right)-\frac{1}{2} \Psi_{\alpha}^{-2} \Psi_{k}^{-2} \rho\left(e_{\alpha}^{2} \lambda_{k}+e_{k}^{2} \lambda_{\alpha}\right) \\
+ & \Psi_{\alpha}^{-2} \Psi_{k}^{-2} \rho\left\{\lambda_{k} e_{\alpha}\left(g\left(\left[e_{\alpha}, e_{k}\right], e_{k}\right)\right)-\lambda_{\alpha} e_{k}\left(g\left(\left[e_{\alpha}, e_{k}\right], e_{\alpha}\right)\right)\right\} \\
+ & {\left[\left\{\frac{1}{2} \Psi_{\alpha}^{-4} e_{\alpha} \lambda_{\alpha}-\Psi_{\alpha}^{-2} \Psi_{k}^{-2} e_{\alpha} \lambda_{k}\right\} g\left(\left[e_{k}, e_{\alpha}\right], e_{k}\right)\right.} \\
& \left.+\left\{\frac{1}{2} \Psi_{k}^{-4} e_{k} \lambda_{k}-\Psi_{\alpha}^{-2} \Psi_{k}^{-2} e_{k} \lambda_{\alpha}\right\} g\left(\left[e_{\alpha}, e_{k}\right], e_{\alpha}\right)\right] .
\end{aligned}
$$




\section{References}

[1] Baider, A., Noncompact Riemannian Manifolds with Discrete Spectra. J. Differ. Geom. 14 (1979), 41-57.

[2] Bishop, R.L. and O'Neill, B., Manifolds of Negative Curvature. Trans. Amer. Math. Soc. 145 (1969), 1-49.

[3] Dieudonné, J., Eléments D’Analyse 1. Gauthier-Villars, 1972.

[4] Eichhorn, J., Riemannsche Mannigfaltigkeiten mit einer zylinderahnlichen Endenmetrik. Math. Nachr. 114 (1983), 23-51.

[5] Gao, L.Z., The Construction of Negatively Ricci Curved Manifolds, Math. Ann. 271 (1985), 185-208.

Department of Mathematics

Kanazawa University

Kanazawa, 920, Japan 